\title{
Monte Carlo simulation on Ebola's disease virus
}

\author{
Zhiyuan Liu ${ }^{\mathrm{a}}$, Qiaobian Xu ${ }^{\mathrm{b}}$, Huiqing Jiang ${ }^{\mathrm{c}}$ \\ School of Energy Power and Mechanical Engineering College, North China Electric Power University, \\ Baoding 071003, China \\ a347435078@qq.com, b1098364642@qq.com, ${ }^{c} 1252698489 @ q q . c o m$
}

Keywords: Ebola epidemic; SIR model; Monte Carlo.

\begin{abstract}
In order to control the spread of the Ebola virus, we use the differential equation and the Monte Carlo algorithm to study the regulation of the spread of the Ebola virus with the intervention of human. We mainly discuss the effect of the contact rate, immune ratio and quarantine ratio to Ebola epidemic. The draw the conclusion that to control the spread of epidemic, reducing the aggregation activities and increasing the immune ratio are effective methods.
\end{abstract}

\section{Introduction}

There is a break of Ebola in Guinea on the middle March and it spread to many countries in western Africa in a short time.By the time August 26, 2014 ,Ebola has killed 1427 people in Sierra Leone, Libya and Guinea. And the dead rates of it is more than 50\%.In order to control the spread of the Ebola virus, we use the differential equation and the Monte Carlo algorithm to study the regulation of the spread of the Ebola virus. We divide the crowd into four groups and modify the original SIR model .Then we build differential equations to describe the regulation. Because the spread of the Ebola virus is a random process, we use Monte Carlo algorithm to simulate the Markov chain process of which the transfer matrix changes over time. Thus, we obtain a regulation that reflects the reality very well.The result shows that the valid contact number and recovery ratio are the key factors to limit the spread of the Ebola virus.

\section{The Differential Equation Model}

To simplify the problem, we make the following hypothesis in the process of modeling:

The total population remains constant (no birth, immigration, emigration and unrelated deaths).

The total population of people used in this model are randomly distributed over area.

The removed can't become infective again.

The incubation period and infectious period of EVD is negligible.

Dead individuals can't transmit disease to susceptible individuals.

We construct a differential equation model to predict the transmission of EVD. It based on the SIR model with the difference that the infected die at a certain rate and susceptible people are likely to become the removed by immunizing with vaccine. The population of a region can be divided into four communities: the susceptible, the infective, the removed and people killed by the virus. The parameters are explained in Table 1.

We have the equation:

$s(t)+i(t)+r(t)+w(t)=1$

According to our assumption, the population of the infected group is reduced in two ways, people can either recover or they are killed by the virus. This can be written as:

$N \frac{d i}{d t}=\lambda i s(1-b) N-a b i N-(1-a) b i N$

The removed group's population is increased by those that recover from the virus added the amount of the susceptible people who become the removed after inoculation. The equation is: 


$$
N \frac{d r}{d t}=a b N i+c N s
$$

The population of the dead group is defined by the number of people that are killed by the virus within the infected group. The equation for the population of the deceased group is written as:

$$
N \frac{d w}{d t}=N i b(1-a)+N(1-b) i
$$

The four equations are presented as:

$$
\begin{aligned}
& \frac{d s}{d t}=-\lambda i s(1-b)-c s-(1-b) i-b i(1-a), s(0)=s_{0} \\
& \frac{d i}{d t}=\lambda(1-b) i s-a b i, i(0)=i_{0} \\
& \frac{d r}{d t}=a b i+c s, r(0)=r_{0} \\
& \frac{d w}{d t}=i b(1-a)+(1-b) i, w(0)=w_{0}
\end{aligned}
$$

Table 1. Notation Parameter Meaning

\begin{tabular}{cc}
\hline Parameter & Meaning \\
\hline $\mathrm{N}$ & The total population of the observed region \\
$\mathrm{i}(\mathrm{t})$ & The susceptible proportion of the total population \\
$\mathrm{r}(\mathrm{t})$ & The infected proportion of the total population \\
$\mathrm{w}(\mathrm{t})$ & The removed proportion of the total population \\
$s_{0}$ & The dead proportion of the total population \\
$i_{0}$ & The initial value of the susceptible \\
$r_{0}$ & The initial value of the infected \\
$w_{0}$ & The initial value of the removed \\
$\lambda$ & The initial value of the dead \\
$\mathrm{a}$ & The number of valid contract by a infected person per unit time \\
$\mathrm{b}$ & The recovery ratio \\
$\mathrm{c}$ & The ratio of the infected quarantined \\
\hline
\end{tabular}

\section{Monte Carlo Algorithm}

In order to improve the model and eliminate the defects which are caused by regarding the parameters as constants, we use the Monte Carlo method to get the transition matrix of the Markov chain and simulate the process. In this problem, the physical state at time $t+1$ depends only on the state at time $t$, so the physical state is a Markov process. We can calculate the transition probability matrix. We use the Monte Carlo method to calculate the transition matrix of the Markov Chain. By this way, we obtain the law about how the Ebola virus transfer in crowd more accurately.

We define the survival time as the period that patients are from infected to dead and it obeys to a uniform distribution of a certain interval. The infected people can only be cured during that time.

We can get some information from the Figure 1:

The infected proportion will increase for a while and then decrease. There appears a peak which indicates that the Ebola virus disease reaches its high point. The model is more realistic to show the situation. Therefore, the medical and health departments should focus on this moment. 
The ratio of the dead begins to increase after the infective line rising for a while. The proportion will continue to rise when the infective reaches the peak point until the ratio of the infective is nearly to zero.

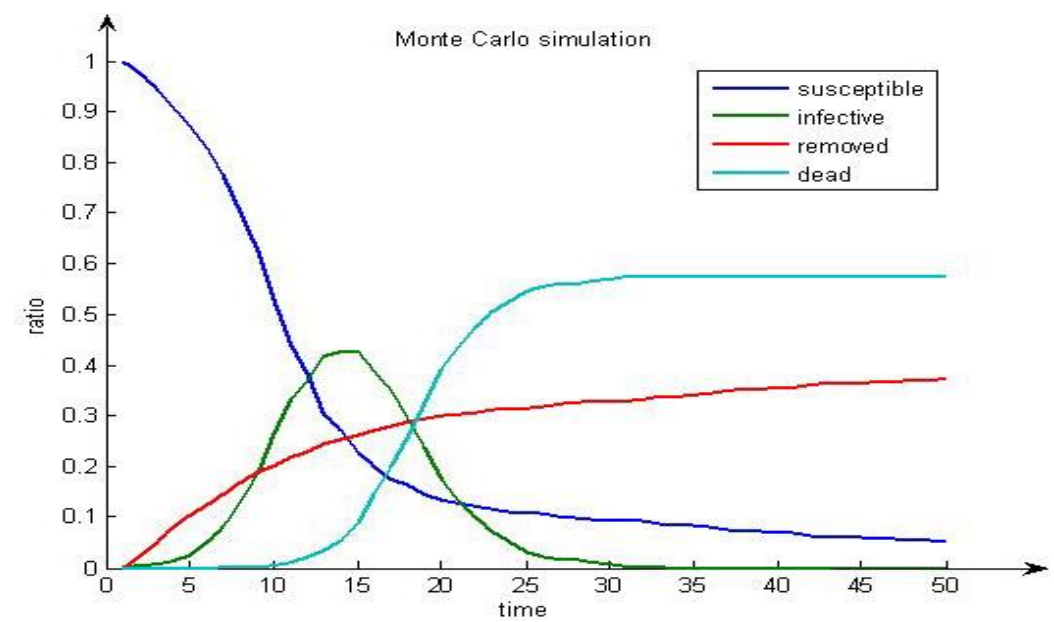

Fig 1. The results of Monte Carlo simulation

\section{Influence Factors}

We know that the initial proportion of the groups and the total population are determined by the observed region.Besides, the removed ratio" $a$ "is decided by the effect of medicine, which is seen as an invariant. we analyze sensitivity by running the program with modified parameters(contract number" $a$ ", the isolation ratio " $b$ "and the immune ratio " $c$ "). The influence on dead rate by changing the value of these parameters are shown in the following figure.

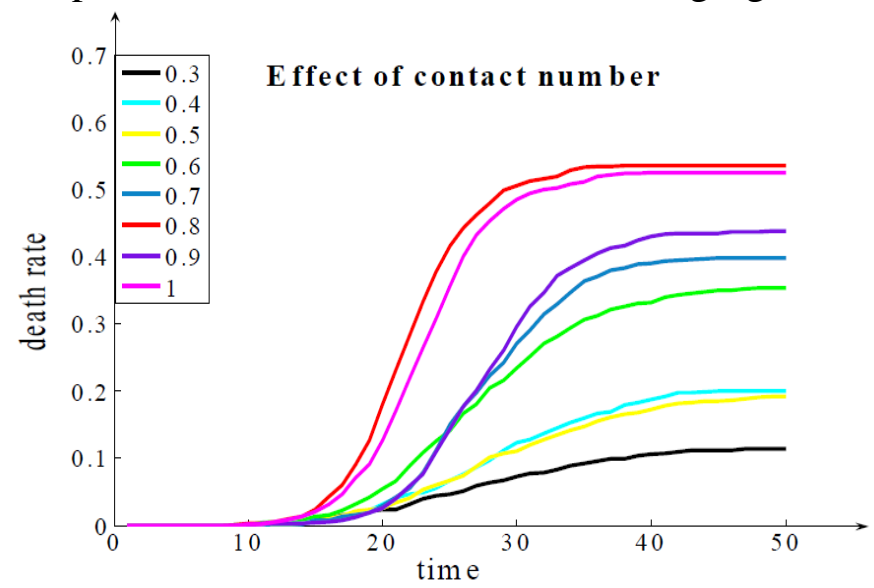

Fig 2. Sensitivity test of contact number

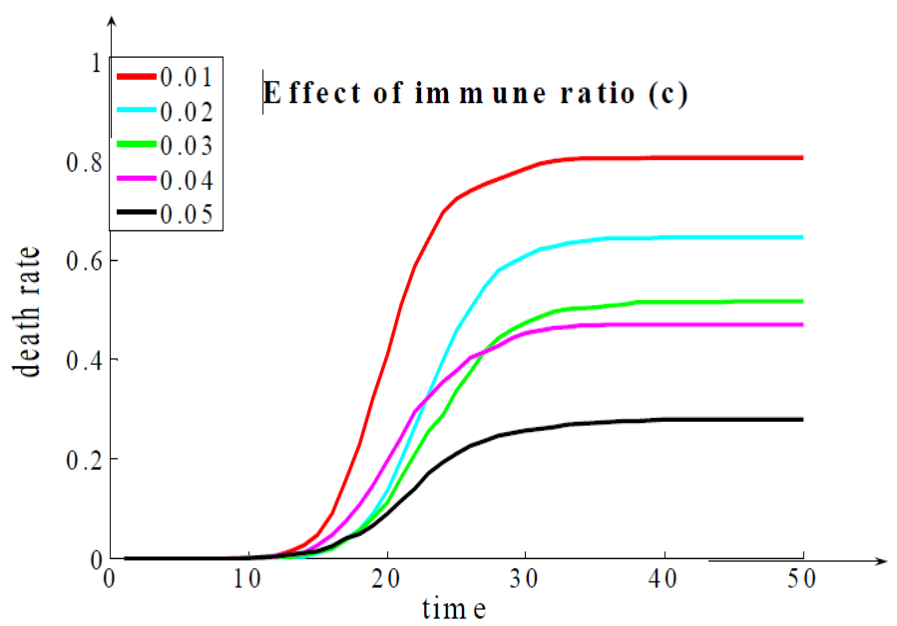

Fig 3. Sensitivity test of immune ratio 


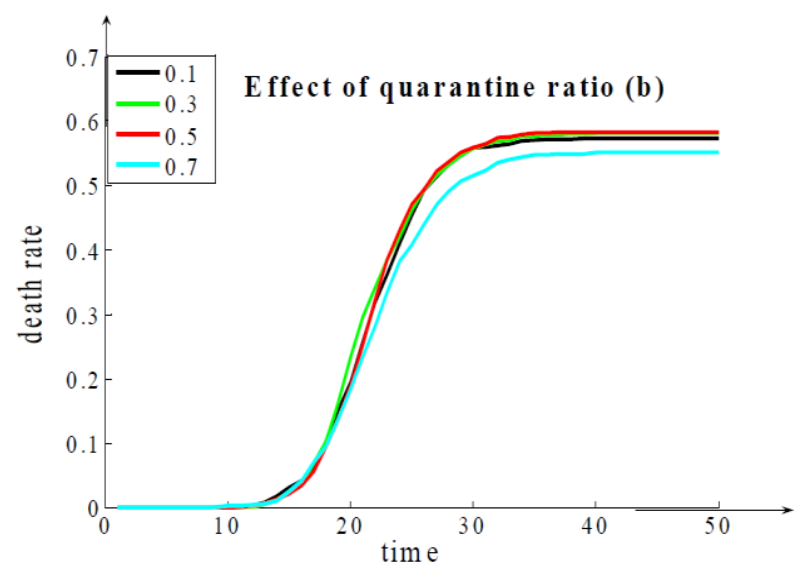

Fig 4. Sensitivity test of quarantine ratio

Figure 2 shows that the dead rate rise when it grows from 0.3 to 1 . The death rate is sensitive to the contact number. This is reasonable, since the value of contact number affects the proportion of the infected significantly.

As we can see in Figure 3, the death rate is sensitive to the immune ratio and to the quarantine ratio, it is insenitive according to Figure 3 .The death rate is insensitive to the quarantine ratio, as we can see in Figure4.

\section{Conclusion}

Monte Carlo is a sensible way to reflect the reality.The population of the infective start to increase to maximum and then decrease with the time going on.To control the spread of epidemic, reducing the aggregation activities and increasing the immune ratio are effective methods.

\section{Reference}

[1]Yarus Z.A Mathematical look at the Ebola Virus[J/OL]. http ://www.home2.fvcc.edu/, 2012. [2]Qiyuan Jiang,Jinxing Xie,Jun Ye. The Mathematical model[M].Beijing:Higher Education Press. [3]ZhiXin Wang.Matlab Program and Mathematical Model Application [M] .Beijing :Science press. [4]World Health Organization. Ebola virus disease(EVD) [EB /OL][2014 -08 -10]: http:/ /www. WHO.int

[5] Ebola virus epidemic in West Africa ,From Wikipedia, the free encyclopedia. http://en. wikipedia.org/wiki/Ebola virus epidemic in West Africa. 